\title{
Clinical utility of a composite scoring system including Charlson Comorbidity Index score in patients with interstitial lung disease
}

\author{
Hiroyuki Yagyu ${ }^{1 \#}$, Kota Murohashi ${ }^{1 \#}$, Yu Hara ${ }^{1}$, Yusuke Saigusa ${ }^{2}$, Ayako Aoki ${ }^{1}$, Nobuaki Kobayashi ${ }^{1}$, \\ Takeshi Kaneko ${ }^{1}$
}

${ }^{1}$ Department of Pulmonology, ${ }^{2}$ Department of Biostatistics, Yokohama City University Graduate School of Medicine, Yokohama, Japan

Contributions: (I) Conception and design: H Yagyu, K Murohashi, Y Hara, Y Saigusa, N Kobayashi, T Kaneko; (II) Administrative support: H Yagyu, K Murohashi, Y Hara; (III) Provision of study materials or patients: H Yagyu, K Murohashi, Y Hara; (IV) Collection and assembly of data: H Yagyu, K Murohashi, Y Hara, A Aoki; (V) Data analysis and interpretation: H Yagyu, K Murohashi, Y Hara, Y Saigusa; (VI) Manuscript writing: All authors; (VII) Final approval of manuscript: All authors.

"These authors contributed equally to this work.

Correspondence to: Yu Hara. Department of Pulmonology, Yokohama City University Graduate School of Medicine, Fukuura, Kanazawa-ku, Yokohama City, 236-0004, Japan. Email: bronchiole0723@yahoo.co.jp.

Background: Prognostic factors have yet to be established for patients with interstitial lung disease (ILD). We aimed to clarify whether the Charlson Comorbidity Index score (CCIS) could help predict disease prognosis in patients with ILD.

Methods: Among ILD patients treated between April 2013 and April 2017, we retrospectively assessed the relationship between baseline clinical parameters including age, sex, CCIS, ILD diagnosis, pulmonary function test results, and 3-year ILD-related events including cause-specific death and first acute exacerbation (AE).

Results: We assessed 180 patients (mean age, 74 years), all of whom underwent pulmonary function testing including percentage predicted diffusion capacity for carbon monoxide $\left(\% \mathrm{D}_{\mathrm{Lco}}\right)$. Underlying pathologies included idiopathic pulmonary fibrosis (IPF) in 57 cases, idiopathic nonspecific interstitial pneumonia (iNSIP) and collagen vascular disease-related interstitial pneumonia in 117 cases, and chronic hypersensitivity pneumonia (CHP) in 6 cases. A composite scoring system comprising IPF diagnosis, CCIS, and \% $\mathrm{D}_{\text {Lco }}$ provided a favorable C-index (0.825) for predicting 3-year ILD-related events. The nomogram for 3-year prognosis revealed the largest contributions from CCIS, $\% \mathrm{D}_{\mathrm{Lco}}$ and IPF diagnosis.

Conclusions: This composite scoring system accounting for IPF diagnosis, CCIS, and $\% \mathrm{D}_{\text {Lco }}$ could provide a useful tool for predicting prognosis in relatively mild ILD patients tolerated to pulmonary diffusion capacity testing.

Keywords: Charlson Comorbidity Index score (CCIS); composite scoring system; diffusion capacity of lung for carbon monoxide; idiopathic pulmonary fibrosis (IPF)

Submitted Mar 13, 2020. Accepted for publication Aug 13, 2020.

doi: $10.21037 /$ jtd-20-1302

View this article at: http://dx.doi.org/10.21037/jtd-20-1302

\section{Introduction}

Clinical biomarkers for predicting the prognosis of interstitial lung disease (ILD) have not been established. An official the American Thoracic Society/the European Respiratory Society/the Japanese Respiratory Society/the
Latin American Thoracic Association (ATS/ERS/JRS/ ALAT) statement proposed various clinical parameters associated with an increased risk of mortality among patients with idiopathic pulmonary fibrosis (IPF) (1). Although these parameters include the severity of dyspnea, 
pulmonary function decline, existence of pulmonary hypertension, desaturation during the 6-min walk test, and the extent of pulmonary involvement on high-resolution computed tomography (HRCT), predicting prognosis using a single parameter appears difficult (2). Actually, prediction of prognosis using composite scoring systems such as GAP [gender (sex) (G), age (A), percentage predicted forced vital capacity (\%FVC) and percentage predicted diffusion capacity of lung for carbon monoxide $\left.\left(\% \mathrm{D}_{\mathrm{Lco}}\right)(\mathrm{P})\right]$ or ILD-GAP has been considered useful in clinical settings $(3,4)$. However, because the parameters used for the GAP or ILD-GAP models are age, sex, ILD diagnosis, and pulmonary function test results, which are based on clinical information derived from only pulmonary involvements, assessment of the presence and severity of comorbidities is also very important for predicting the prognosis of ILD patients (5).

The Charlson Comorbidity Index score (CCIS) is a summed score of 19 comorbidities weighted according to severity (6). The CCIS was developed to assess risk of death from comorbidities and has been widely applied as a prognostic indicator for patients with colorectal cancer, advanced non-small cell lung carcinoma and acute myocardial infarction (7-9). In our previous report on CCIS, serum lactate dehydrogenase (LDH) and sex were useful for predicting the prognosis of patients with acute exacerbation (AE) of ILD (10). However, whether these characteristics are useful for predicting prognosis in patients with ILD in a stable condition remains unclear.

The present study retrospectively investigated clinical parameters (including sex, age, ILD diagnosis, pulmonary function test, and CCIS) that could help predict prognosis in patients with ILD in a stable condition. We also attempted to construct a scoring system and to construct a nomogram from that scoring system that could predict 3-year ILD-related events, including cause-specific death or first AE.

\section{Methods}

This retrospective, observational study was performed using data from patients treated at Yokohama City University Hospital between April 2013 and April 2017. The medical records of all patients with ILD who met the following inclusion criteria were reviewed: stable ILD cases including IPF, idiopathic nonspecific interstitial pneumonia (iNSIP), chronic hypersensitivity pneumonia (CHP), or collagen vascular diseases-related interstitial pneumonia (CVD-IP); and availability of pulmonary function test results including DLco (Figure 1). We defined the stable ILD cases as patients who required only follow-up without specific treatments including oral corticosteroid or anti-fibrotic agents and had not experienced acute respiratory deterioration including AE previously. Other ILD patients including pulmonary sarcoidosis, cryptogenic organizing pneumonia, drug or radiation induced lung injuries, and other unclassified ILDs were excluded. We assessed the relationship between baseline clinical parameters including age, sex, CCIS, ILD diagnosis, pulmonary function test results, and 3-year ILDrelated events including cause-specific death or first $\mathrm{AE}$. We collected 3-year ILD-related events mainly from medical records. For patients who did not die in our hospital, the disease outcomes were confirmed by telephone. In addition, only one patient $(0.5 \%)$ transferred to another hospital for the purpose of best supportive case due to severe deterioration of respiratory status was lost of follow-up, therefore we decided the transfer date as the decision date of disease outcome.

A diagnosis of idiopathic interstitial pneumonia (IIP) was confirmed by physical findings, serological testing, findings from HRCT, and lung biopsy specimens, based on the official statement for IIP $(1,11)$. However, patients from whom a lung biopsy could not be obtained were diagnosed based on the radiological classification $(1,11)$. The diagnosis of CVD-IP was confirmed by physical findings, serological testing, and HRCT findings consistent with ILD. Histological evaluation of lung biopsy specimens was undertaken to exclude other specific diseases. The event of AE of ILD was defined as: unexplained worsening of dyspnea; hypoxemia or severely impaired gas exchange; new alveolar infiltrates on radiography; and absence of an alternative explanation such as infection, pulmonary embolism, pneumothorax, or heart failure (12-14).

\section{Statistical analysis}

Data were statistically analyzed using JMP12 (SAS Institute, Cary, NC, USA) and R software, version 3.5.1 (The R Foundation for Statistical Computing, Vienna, Austria), and are expressed as mean \pm standard deviation. Groups were compared using Wilcoxon rank-sum tests. Optimal parameter cut-off values were determined by Youden index for receiver operator characteristics curves. Predictors of 3-year ILD-related events were investigated by backward stepwise algorithm, forcing the diagnosis of IPF (which has a significant impact on clinical prognosis) 
Table 1 Patient's characteristics

\begin{tabular}{|c|c|c|c|c|c|}
\hline Characteristics & Overall cases & IPF & CVD-IP/iNSIP & $\mathrm{CHP}$ & $P$ value \\
\hline Age, years & $71.9 \pm 9.2$ & $73.3 \pm 7.2$ & $71.4 \pm 9.2$ & $67.8 \pm 21.3$ & 0.425 \\
\hline Male sex, n (\%) & $119[66]$ & $49[86]$ & $66[56]$ & 4 [67] & $<0.001$ \\
\hline CCIS & $2.5 \pm 2.2$ & $2.6 \pm 2.0$ & $2.5 \pm 2.2$ & $1.5 \pm 1.2$ & 0.418 \\
\hline FVC, \%predicted & $93.9 \pm 18.7$ & $93.5 \pm 18.5$ & $94.1 \pm 19.2$ & $92.8 \pm 10.8$ & 0.993 \\
\hline $\mathrm{D}_{\text {Lco }}, \%$ predicted & $91.7 \pm 30.2$ & $80.5 \pm 28.2$ & $97.6 \pm 30.2$ & $83.1 \pm 17.6$ & 0.002 \\
\hline \multicolumn{6}{|l|}{ Outcome } \\
\hline Follow-up, days & $687 \pm 337$ & $649 \pm 327$ & $701 \pm 344$ & $775 \pm 294$ & 0.514 \\
\hline
\end{tabular}

Incidence of events included ILD-related death or first acute exacerbation of ILD. CCIS, Charlson Comorbidity Index; CHP, chronic hypersensitivity pneumonia; CVD-IP, collagen vascular disease-related interstitial pneumonia; DLco, lung diffusing capacity for carbon monoxide; FVC, forced vital capacity; iNSIP, idiopathic nonspecific interstitial pneumonia; ILD, interstitial lung disease; IPF, idiopathic pulmonary fibrosis.

into the predictors, and using 5-fold cross-validated Uno's concordance index (C-index) (15). C-indices are reported as the mean value calculated from 100,000 bootstrap samples. A nomogram was established based on the scoring systems to predict 3-year ILD-related events. When comparing 3-year ILD-related events including cause-specific death or first $\mathrm{AE}$ among groups depending on the scoring system, Kaplan-Meier curves and adjusted survival curves were employed. Log-rank and stratified log-rank testing were also performed with strata based on the identified predictors. Values of $\mathrm{P}<0.05$ were considered significant.

\section{Ethics approval}

The institutional review board at Yokohama City University Hospital approved this study (approval number B190300005). In all patients, we waived informed consent based on the retrospective design and provided information on the opt-out process (https://www.yokohama-cu.ac.jp/ amedrc/ethics/ethical/fuzoku_optout.html). The study was conducted in accordance with the Declaration of Helsinki (as revised in 2013).

\section{Results}

\section{Patient characteristics}

Table 1 shows the clinical characteristics of the 180 patients evaluated, including 57 patients with IPF and 123 patients with non-IPF (idiopathic NSIP and CVD-IP, n=117; CHP, n=6). CVD-IP included rheumatoid arthritis, antineutrophil cytoplasmic antibody-associated vasculitis, polymyositis/dermatomyositis, and Sjögren syndrome. No significant differences were identified in clinical parameters other than sex or $\% \mathrm{D}_{\mathrm{Lco}}$.

\section{Accuracy of composite parameters in predicting 3-year ILD-related events}

To investigate the accuracy of the scoring system for 3-year ILD-related events, we calculated C-index for the combination of various clinical parameters including age, sex, CCIS, IPF diagnosis, and pulmonary function test results. The composite scoring system including IPF diagnosis, CCIS, and $\% \mathrm{D}_{\mathrm{Lco}}$ offered a higher $\mathrm{C}$-index than single predictors or any other combination of predictors (Table 2). The three independent risk factors were selected according to appellate statistical criteria. We prepared a nomogram based on these variables (Figure 2). Each selected indicator was assigned a corresponding score according to its own value on the nomogram. The calculated total score provided a predicted 3-year ILD-related event-free survival rate. By determining the interval in which the total score is located, the 3-year ILD-related event-free survival probability can be obtained more simply. 


\section{Composite scoring system for predicting 3-year ILD-} related events

The composite scoring system including IPF diagnosis, CCIS, and $\% \mathrm{D}_{\mathrm{Lco}}$ represents the global score (ranging between 0 and 6 ) calculated from the three clinical parameters: CCIS $(0-1,1 ; 2-3,2 ; 4-5,3 ; \geq 6,4)$; IPF diagnosis (non-IPF, 0 ; IPF, 1); and $\% \mathrm{D}_{\text {Lco }}(\geq 75.1,0 ; \leq 75.1,1)$

Table 2 Accuracy of scoring system for predicting 3-year events including ILD-related death or first acute exacerbation of ILD

\begin{tabular}{lc}
\hline Variable & C-index \\
\hline CCIS & 0.747 \\
IPF + CCIS + FVC & 0.799 \\
IPF + CCIS + DLco & 0.825 \\
IPF + Age + Sex + CCIS + DLco & 0.792 \\
IPF + Age + Sex + CCIS + FVC & 0.778 \\
IPF + Age + Sex + FVC + DLco & 0.671 \\
IPF + Age + Sex + CCIS + FVC + DLco & 0.788 \\
\hline
\end{tabular}

$\mathrm{C}$-indices are reported as the mean value calculated from 100,000 bootstrap samples. CCIS, Charlson Comorbidity Index score; DLco, lung diffusing capacity for carbon monoxide; FVC, forced vital capacity; ILD, interstitial lung disease; IPF, idiopathic pulmonary fibrosis.
(Table 3). We compared patients with ILD according to the composite scoring system (score $\leq 2 v s$. $\geq 3$ or score $\leq 2 v s .=3$ vs. 24 ) (Figures 3,4). Both log-rank and stratified log-rank tests showed that Kaplan-Meier survival curves of these groups differed significantly $(\mathrm{P}<0.001)$.

\section{Relationship between CCIS and incidence of 3-year ILD- related events}

Figure 5 shows the relationship between CCIS and 3-year ILD-related event. The frequency of 3-year ILD-related events tended to increase with increasing CCIS, and this tendency was more pronounced with higher composite scores $(\geq 3)$.

\section{Discussion}

In patients with ILD, for which the clinical course varies considerably in severity and rate of progression, prognosis is difficult to predict using established single biomarkers, such as age, level of dyspnea, $\mathrm{D}_{\mathrm{Lco}}$, oxygen desaturation during the 6-min walk test, extent of honeycombing on HRCT images, and pulmonary hypertension $>25 \mathrm{mmHg}$ at rest (1). Composite approaches have been developed using peripheral blood parameters, physiological parameters, and radiographic parameters to provide more accurate

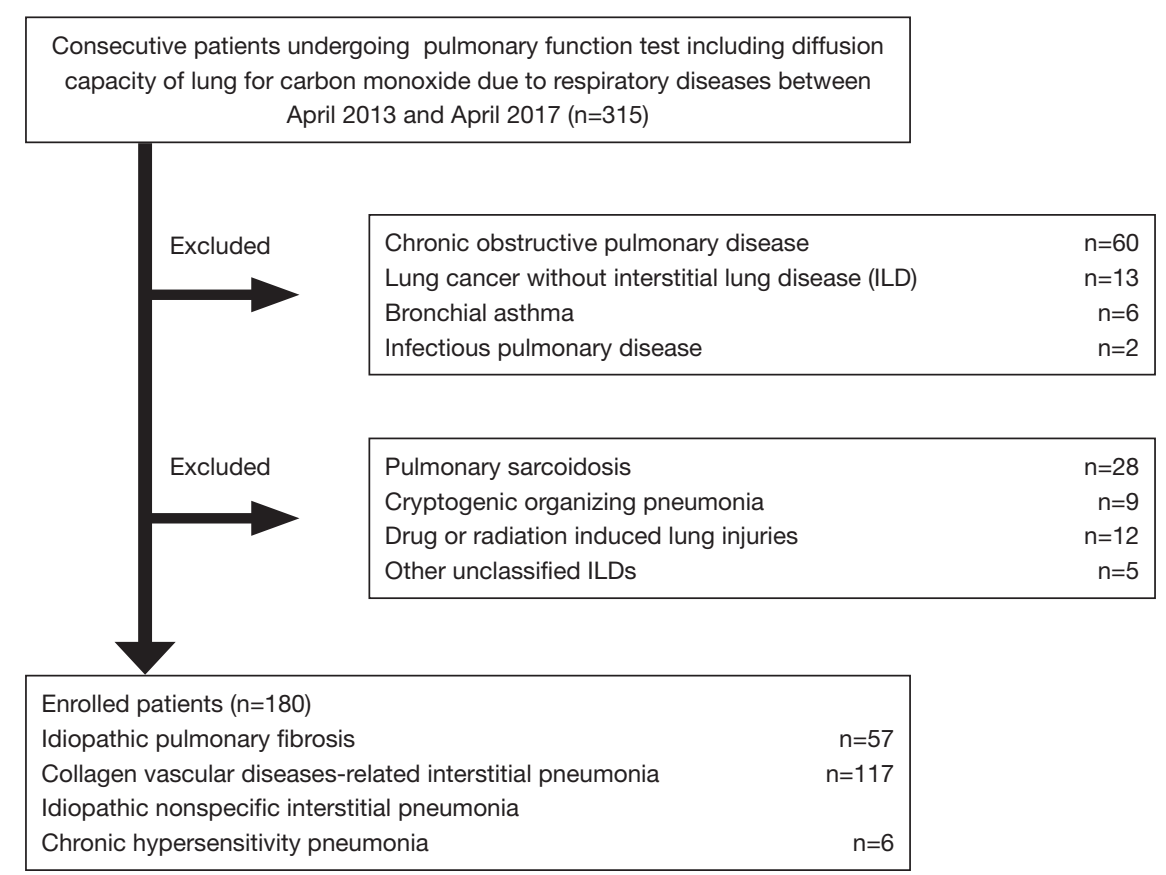

Figure 1 Flowchart of the participants selection process. 


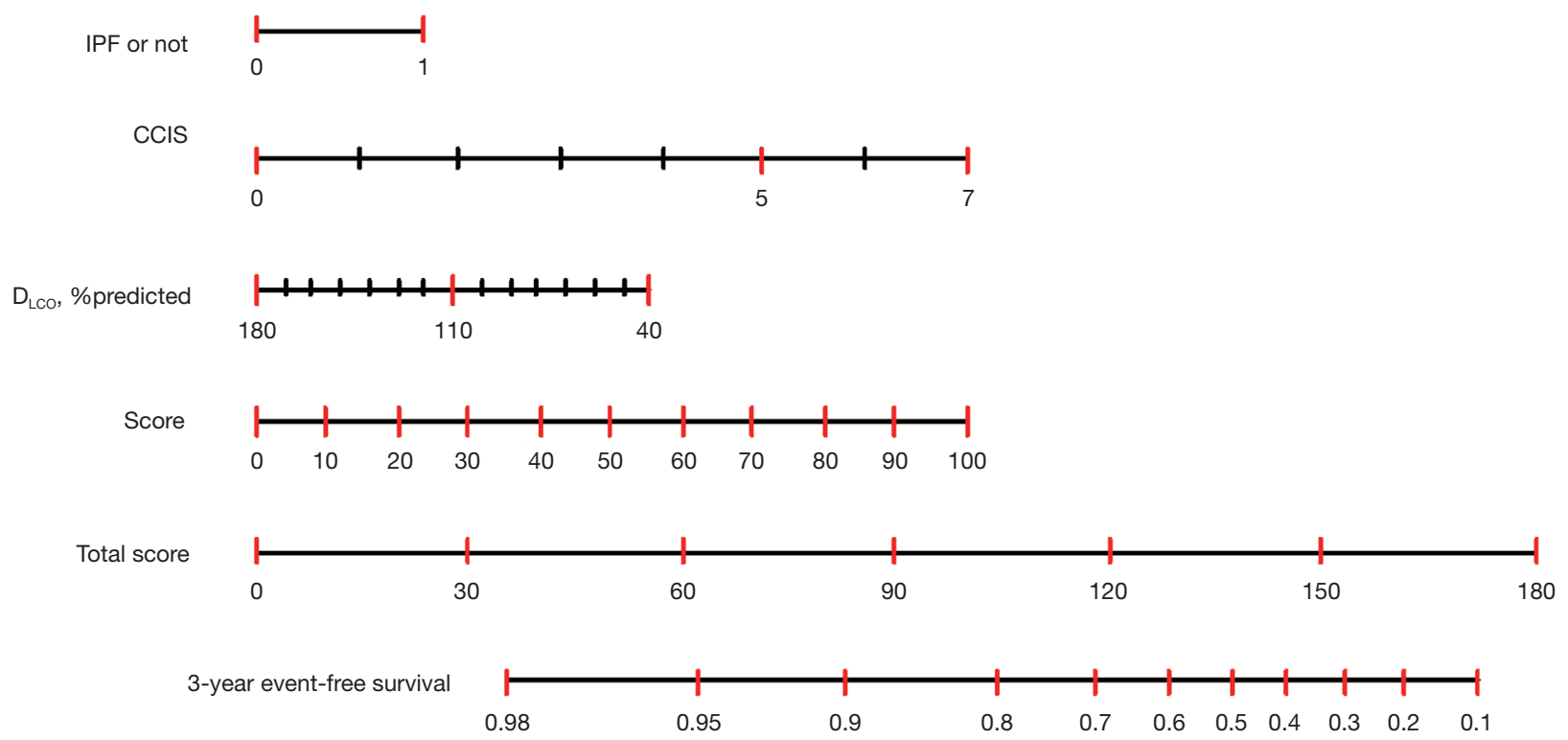

Figure 2 The nomogram for predicting 3-year ILD-related events. Draw a line perpendicular from the corresponding axis of each risk factor until it reaches the top line labeled "Points". Total the number of points for all risk factors, then draw a line descending vertically from the axis labeled "Total score" until it intercepts the survival axis to determine the 3 -year event-free survival. For binary variables, $0=$ no and $1=$ yes. CCIS, Charlson Comorbidity Index score; IPF, idiopathic pulmonary fibrosis; $\mathrm{D}_{\mathrm{Lco}}$, diffusion capacity for carbon monoxide.

Table 3 Scoring system including IPF diagnosis, CCIS, DLco

\begin{tabular}{lcc}
\hline Predictors & Details & Score \\
\hline The diagnosis & IPF & 1 \\
& Not IPF & 0 \\
CCIS & $\geq 6$ & 4 \\
& $4-5$ & 3 \\
& $2-3$ & 2 \\
& $0-1$ & 1 \\
DLco, \%predicted & $<75.1$ & 1 \\
& $\geq 75.1$ & 0 \\
\hline
\end{tabular}

CCIS, Charlson Comorbidity Index score; $\mathrm{D}_{\text {Lco }}$, lung diffusion capacity for carbon monoxide; IPF, idiopathic pulmonary fibrosis.

prognostic information. We have recently applied prognostic modeling using the GAP scoring system in patients with IPF, and the ILD-GAP scoring system in patients with fibrosing ILD $(3,4)$. However, Lee et al. reported that prognosis in IPF patients with a GAP score of 3 differed significantly from that in other stage I groups and stage II groups of Asian patients (16). Furthermore, the high frequencies of comorbidities including gastroesophageal reflex disease, heart failure, coronary artery disease, stroke, and cancer are increasingly being recognized as impacting prognosis in patients with ILD (5). Therefore, the present research established a prognostic scoring system and a nomogram to predict 3-year survival by adding CCIS, which is not directly related to lung involvement, as well as ILD diagnosis, age, sex, and pulmonary function test results.

Pulmonary function test results have been proposed as a major prognostic determinant in patients with $\operatorname{ILD}(3,4,17)$. Six-month changes in FVC provide a significant predictor of survival in patients with fibrotic interstitial pneumonia, while baseline FVC is of unclear predictive value (18). Recent evidence-based guidelines for the management of IPF state that an absolute decline in FVC $\geq 10 \%$ over time is an acceptable marker of disease progression and can be used to estimate risk of future mortality in patients with IPF (19). $\mathrm{D}_{\mathrm{Lco}}$ is more reliably predictive of survival at baseline, and a threshold of approximately $35 \%$ has been associated with increased risk of mortality (20). Mortality was also substantially higher in those with significant (i.e., $>15 \%$ ) deterioration in gas transfer on follow-up at 12 months (21). In the present research, C-index derived from the 

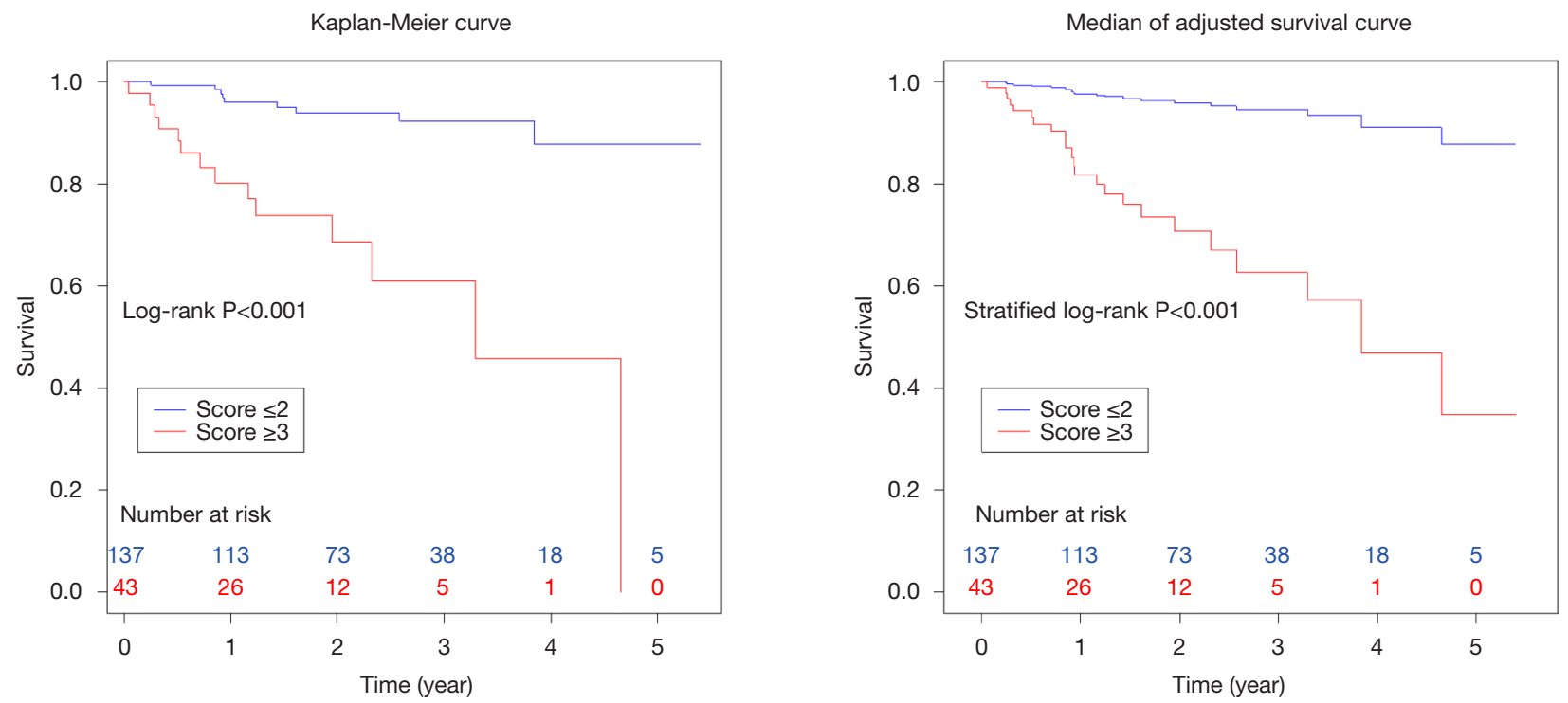

Figure 3 Comparison of patients with ILD according to the composite scoring system including ILD diagnosis, CCIS, and \% $\mathrm{D}_{\text {Lco }}$ (score $\leq 2$ $v s . \geq 3$ ). The composite score is the global score (ranging between 0 and 6 ) calculated from the three parameters: CCIS (0-1, 1; 2-3, 2; 4-5; $3 ; \geq 6,4)$; ILD diagnosis (non-IPF, 0 ; IPF, 1 ); and $\% \mathrm{D}_{\mathrm{Lco}}(\geq 75.1,0 ;<75.1,1)$. Patients are categorized into two groups based on composite score: score $\leq 2 v s . \geq 3$. Three-year ILD-related event-free survival rates are significantly better in the former group ( $\mathrm{n}=137$ ) than latter group ( $\mathrm{n}=43 ; \mathrm{P}<0.001$ ) (A: log-rank test; B: stratified log-rank test). CCIS, Charlson Comorbidity Index score; ILD, interstitial lung disease; IPF, idiopathic pulmonary fibrosis; $\mathrm{D}_{\mathrm{Lco}}$, diffusion capacity for carbon monoxide.
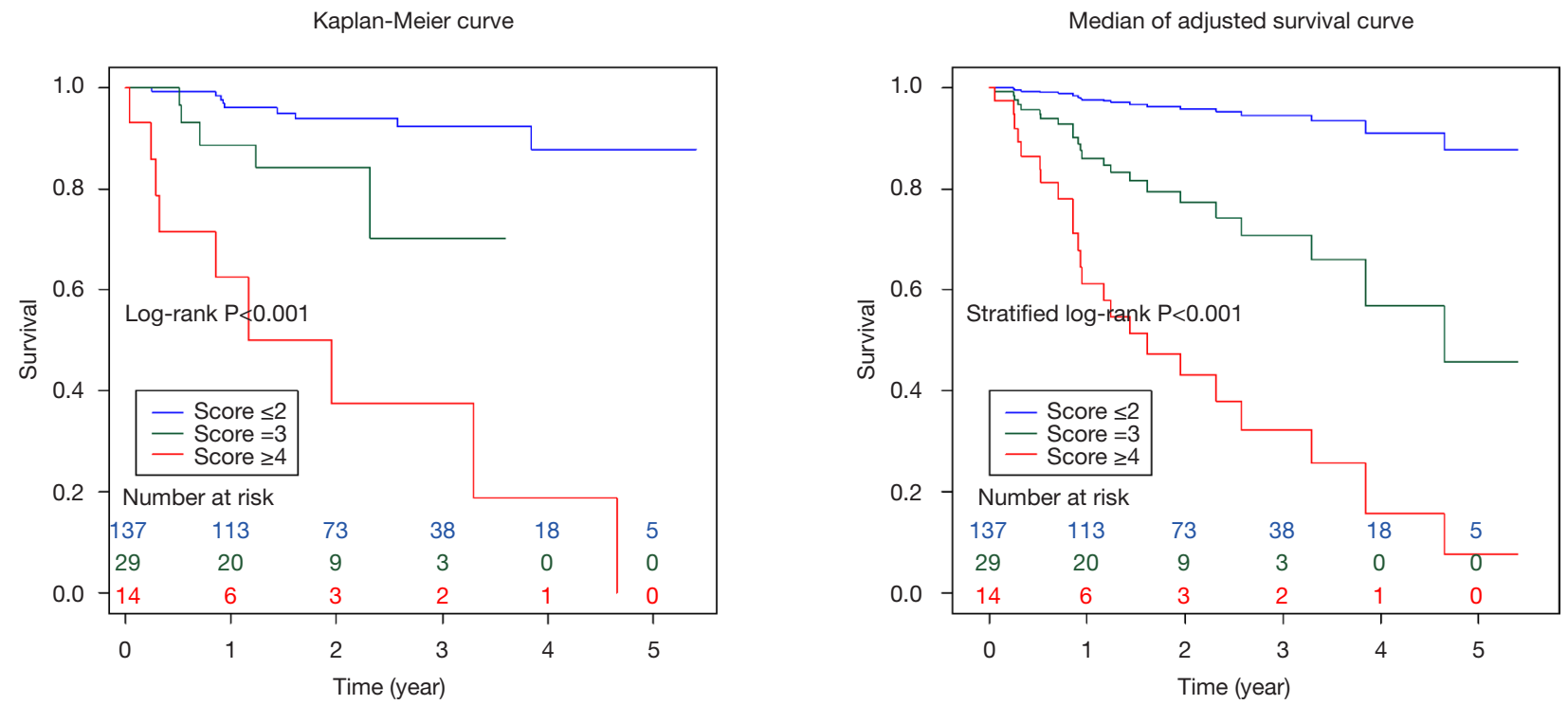

Figure 4 Comparison of patients with ILD according to the composite scoring system including ILD diagnosis, CCIS, and \% $\mathrm{D}_{\mathrm{Lco}}$ (score $\leq 2 v s .=3 v s . \geq 4)$. We categorized the patients having the composite score ( $\geq 3$; the latter group in Figure 3) into two groups: score $=3$ vs. $\geq 4$. Then we compared three-year ILD-related event-free survival rates between three groups: score $\leq 2(\mathrm{n}=137) v s .=3$ ( $\mathrm{n}=29) v s . \geq 4(\mathrm{n}=14)$. Both log-rank and stratified log-rank tests showed that Kaplan-Meier survival curves of these groups differed significantly $(\mathrm{P}<0.001)(\mathrm{A}$ : log-rank test; B: stratified log-rank test). CCIS, Charlson Comorbidity Index score; ILD, interstitial lung disease; $\mathrm{D}_{\text {Lco, }}$ diffusion capacity for carbon monoxide. 


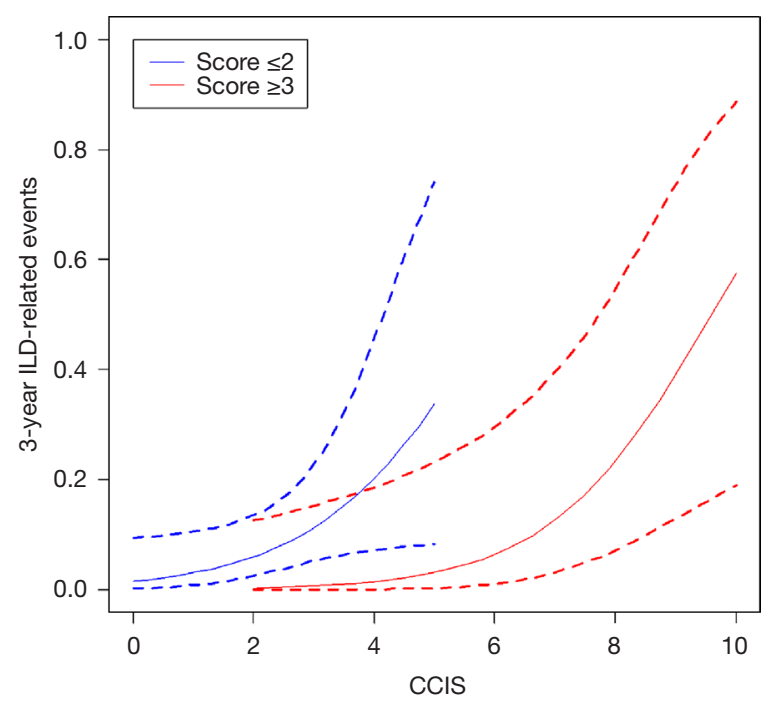

Figure 5 Relationship between CCIS and incidence of 3-year ILD-related events. Frequency of 3-year ILD-related events increases with increasing CCIS, with a more pronounced tendency for higher composite scores $(\geq 3)$. Dashed lines indicate $95 \%$ confidence intervals. CCIS, Charlson Comorbidity Index score; ILD, interstitial lung disease.

combination of $\mathrm{D}_{\mathrm{Lco}}$ and other parameters was higher than that from \% FVC or other parameters. Given the above, baseline $\% \mathrm{D}_{\mathrm{Lco}}$ was expected to be more important than $\% \mathrm{FVC}$ in predicting 3 -year ILD-related events.

Evaluation of comorbidities appears important in determining the prognosis of ILD. Kreuter et al. reported significant negative impacts of arteriosclerosis, other cardiovascular diseases (mainly valvular heart disease, cardiac arrhythmias, and dilated cardiomyopathy), and lung cancer comorbidities on survival among patients with IPF (5). In addition, other reports have identified cardiovascular disease, congestive heart failure, and lung cancer as significant predictors for $\mathrm{AE}$ onset and prognosis (22). In a cohort study that included 1,285 patients with ILD conducted by The CAnadian REgistry for Pulmonary Fibrosis (CARE-PF), CCIS was similar across ILDs and ranged from a mean of $1.2 \pm 0.9$ in non-IPF IIPs to $2.1 \pm 1.0$ in CVD-IP (23). In the present research, we found that similar to previous cohort studies, CCIS did not differ significantly across ILDs and could represent a strong prognostic predictor in patients with ILDs. Interestingly, the frequency of 3-year ILD-related events tended to increase with increasing CCIS, and this tendency was more pronounced with higher composite scores $(\geq 3)$. This result suggested that our composite scoring including CCIS could accurately predict 3-year ILD-related events. A further study is needed to prove if the management of comorbidities improves the prognosis of ILD.

This retrospective study of a small number of patients from only one institution has some limitations. To verify the utility and reproducibility of this composite scoring system, large-scale, multi-institutional prospective collaborative research is essential. The majority of patients enrolled in this study were not so severely ill that pulmonary function tests including $\mathrm{D}_{\mathrm{Lco}}$ could be tolerated, so this point suggested a possible source of bias for this study. Therefore, the results in this study should be applied to the mild ILD patients excluding severe cases who are not tolerant to pulmonary diffusion capacity testing. On the other hand, as with the C-index of IPF+CCIS+\%DLco, that of $\mathrm{IPF}+\mathrm{CCIS}+\% \mathrm{FVC}$ was relatively acceptable. Actually, it was found that the higher the composite scoring system using ILD diagnosis, CCIS, and \% FVC, the higher the frequency of 3-year ILD-related events (Table S1, Figures $S 1, S 2$ ). In severe cases that are not well tolerated, it may be more practical to evaluate with $\% \mathrm{FVC}$, which can be performed more easily, however the future validation targeting all ILD patients ranging from mild to severe cases is necessary.

In conclusion, we found that CCIS, $\% \mathrm{D}_{\mathrm{Lco}}$, and IPF diagnosis offer significant predictors of ILD events among patients with stable ILD.

\section{Acknowledgments}

Funding: None.

\section{Footnote}

Data Sharing Statement: Available at http://dx.doi. org/10.21037/jtd-20-1302

Conflicts of Interest: All authors have completed the ICMJE uniform disclosure form (available at http://dx.doi. org/10.21037/jtd-20-1302). The authors have no conflicts of interest to declare.

Ethical Statement: The authors are accountable for all aspects of the work in ensuring that questions related to the accuracy or integrity of any part of the work are appropriately investigated and resolved. The study was conducted in accordance with the Declaration of Helsinki (as revised in 2013). The institutional review board at 
Yokohama City University Hospital approved this study (approval number B190300005). In all patients, we waived informed consent based on the retrospective design and provided information on the opt-out process (https://www. yokohama-cu.ac.jp/amedrc/ethics/ethical/fuzoku_optout. html).

Open Access Statement: This is an Open Access article distributed in accordance with the Creative Commons Attribution-NonCommercial-NoDerivs 4.0 International License (CC BY-NC-ND 4.0), which permits the noncommercial replication and distribution of the article with the strict proviso that no changes or edits are made and the original work is properly cited (including links to both the formal publication through the relevant DOI and the license). See: https://creativecommons.org/licenses/by-nc-nd/4.0/.

\section{References}

1. Raghu G, Collard HR, Egan JJ, et al. ATS/ERS/JRS/ ALAT Committee on Idiopathic Pulmonary Fibrosis. An official ATS/ERS/JRS/ALAT statement: idiopathic pulmonary fibrosis: evidence-based guidelines for diagnosis and management. Am J Respir Crit Care Med 2011;183:788-824.

2. Hara $Y$, Shinkai M, Rubin BK. Biomarkers for staging and evaluating the therapy for idiopathic pulmonary fibrosis. Clin Pulm Med 2015;22:165-71.

3. Ley B, Ryerson CJ, Vittinghoff E, et al. A multidimensional index and staging system for idiopathic pulmonary fibrosis. Ann Intern Med 2012;156:684-91.

4. Ryerson CJ, Vittinghoff E, Ley B, et al. Predicting survival across chronic interstitial lung disease: the ILD-GAP model. Chest 2014;145:723-8.

5. Kreuter M, Ehlers-Tenenbaum S, Palmowski K, et al. Impact of Comorbidities on Mortality in Patients with Idiopathic Pulmonary Fibrosis. PLoS One 2016;11:e0151425.

6. Charlson ME, Pompei P, Ales KL, et al. A new method of classifying prognostic comorbidity in longitudinal studies: development and validation. J Chronic Dis 1987;40:373-83.

7. Tominaga T, Nonaka T, Takeshita H, et al. The Charlson Comorbidity Index as an Independent Prognostic Factor in Older Colorectal Cancer Patients. Indian J Surg 2018;80:54-60.

8. Zhao L, Leung LH, Wang J, et al. Association between Charlson comorbidity index score and outcome in patients with stage IIIB-IV non-small cell lung cancer. BMC Pulm
Med 2017;17:112.

9. Núñez JE, Núñez E, Fácila L, et al. Prognostic value of Charlson comorbidity index at 30 days and 1 year after acute myocardial infarction. Rev Esp Cardiol 2004;57:842-9.

10. Murohashi K, Hara $Y$, Saigusa $Y$, et al. Clinical significance of Charlson Comorbidity Index as a prognostic parameter for patients with acute or subacute idiopathic interstitial pneumonias and acute exacerbation of collagen vascular diseases-related interstitial pneumonia. J Thorac Dis 2019;11:2448-57.

11. Travis WD, Costabel U, Hansell DM, et al. ATS/ ERS Committee on Idiopathic Interstitial Pneumonias. An official American Thoracic Society/European Respiratory Society statement: Update of the international multidisciplinary classification of the idiopathic interstitial pneumonias. Am J Respir Crit Care Med 2013;188:733-48.

12. Collard HR, Moore BB, Flaherty KR, et al. Idiopathic Pulmonary Fibrosis Clinical Research Network Investigators. Acute exacerbations of idiopathic pulmonary fibrosis. Am J Respir Crit Care Med 2007;176:636-43.

13. Park IN, Kim DS, Shim TS, et al. Acute exacerbation of interstitial pneumonia other than idiopathic pulmonary fibrosis. Chest 2007;132:214-20.

14. Tachikawa $\mathrm{R}$, Tomii $\mathrm{K}$, Ueda $\mathrm{H}$, et al. Clinical features and outcome of acute exacerbation of interstitial pneumonia: collagen vascular diseases-related versus idiopathic. Respiration 2012;83:20-7.

15. Uno H, Cai T, Pencina MJ, et al. On the C-statistics for evaluating overall adequacy of risk prediction procedures with censored survival data. Stat Med 2011;30:1105-17.

16. Lee SH, Kim SY, Kim DS, et al. Predicting survival of patients with idiopathic pulmonary fibrosis using GAP score: a nationwide cohort study. Respir Res 2016;17:131.

17. Yıldırım F, Türk M, Bitik B, et al. Comparison of clinical courses and mortality of connective tissue diseaseassociated interstitial pneumonias and chronic fibrosing idiopathic interstitial pneumonias. Kaohsiung J Med Sci 2019;35:365-72.

18. Collard HR, King TE Jr, Bartelson BB, et al. Changes in clinical and physiologic variables predict survival in idiopathic pulmonary fibrosis. Am J Respir Crit Care Med 2003;168:538-42.

19. Flaherty KR, Mumford JA, Murray S, et al. Prognostic implications of physiologic and radiographic changes in idiopathic interstitial pneumonia. Am J Respir Crit Care Med 2003;168:543-8.

20. Jegal Y, Kim DS, Shim TS, et al. Physiology is a stronger 
predictor of survival than pathology in fibrotic interstitial pneumonia. Am J Respir Crit Care Med 2005;171:639-44.

21. Latsi PI, du Bois RM, Nicholson AG, et al. Fibrotic idiopathic interstitial pneumonia: the prognostic value of longitudinal functional trends. Am J Respir Crit Care Med 2003;168:531-7.

Cite this article as: Yagyu H, Murohashi K, Hara Y, Saigusa Y, Aoki A, Kobayashi N, Kaneko T. Clinical utility of a composite scoring system including Charlson Comorbidity Index score in patients with interstitial lung disease. J Thorac Dis 2020;12(10):5774-5782. doi: 10.21037/jtd-20-1302
22. Kakugawa T, Sakamoto N, Sato S, et al. Risk factors for an acute exacerbation of idiopathic pulmonary fibrosis. Respir Res 2016;17:79.

23. Fisher JH, Kolb M, Algamdi M, et al. Baseline characteristics and comorbidities in the CAnadian REgistry for Pulmonary Fibrosis. BMC Pulm Med 2019;19:223. 
Kaplan-Meier curve

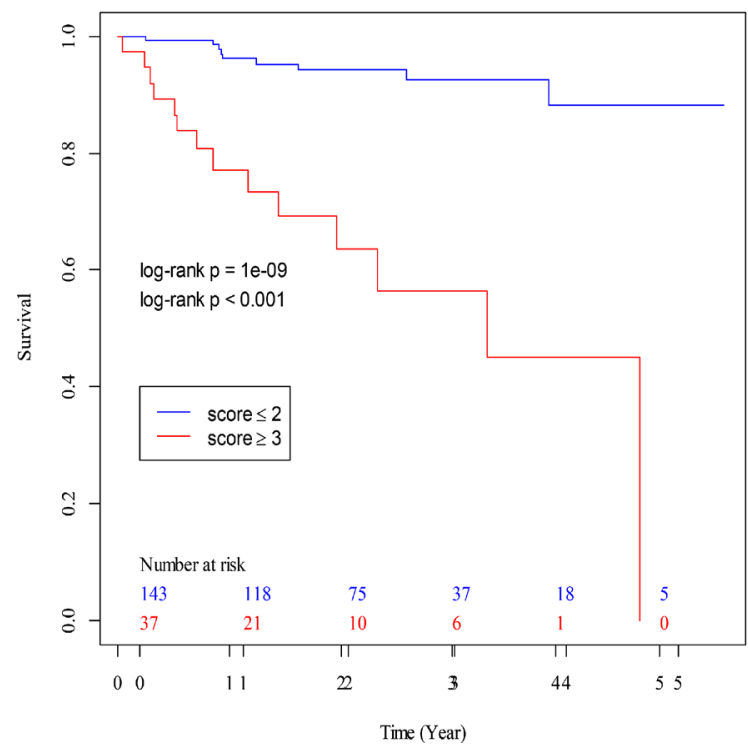

Median of adjusted survival curve

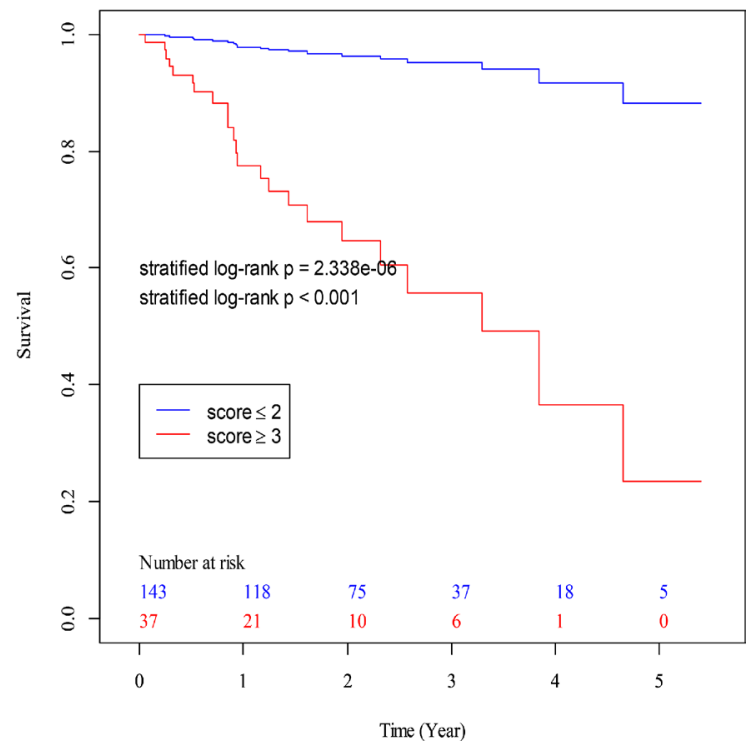

Figure S1 Comparison of patients with ILD according to composite scoring system using ILD diagnosis, CCIS, and \%FVC (score $\leq 2 v s$. $\geq 3$ ). The composite score is the global score (ranging between 0 and 6 ) calculated from the three parameters: CCIS $(0-1,1 ; 2-3,2 ; 4-5$; $3 ; \geq 6,4)$; ILD diagnosis (non-IPF, 0 ; IPF, 1); and $\% \mathrm{FVC}(\geq 70.0,0 ;<70.0,1)$. Patients are categorized into two groups based on composite score: score $\leq 2 v s . \geq 3$. Three-year ILD-related event-free survival rates are significantly better in the former group (n=143) than latter group $(\mathrm{n}=37 ; \mathrm{P}<0.001)$ (A: log-rank test; B: stratified log-rank test). CCIS, Charlson Comorbidity Index score; ILD, interstitial lung disease; IPF, idiopathic pulmonary fibrosis; FVC, forced vital capacity. 

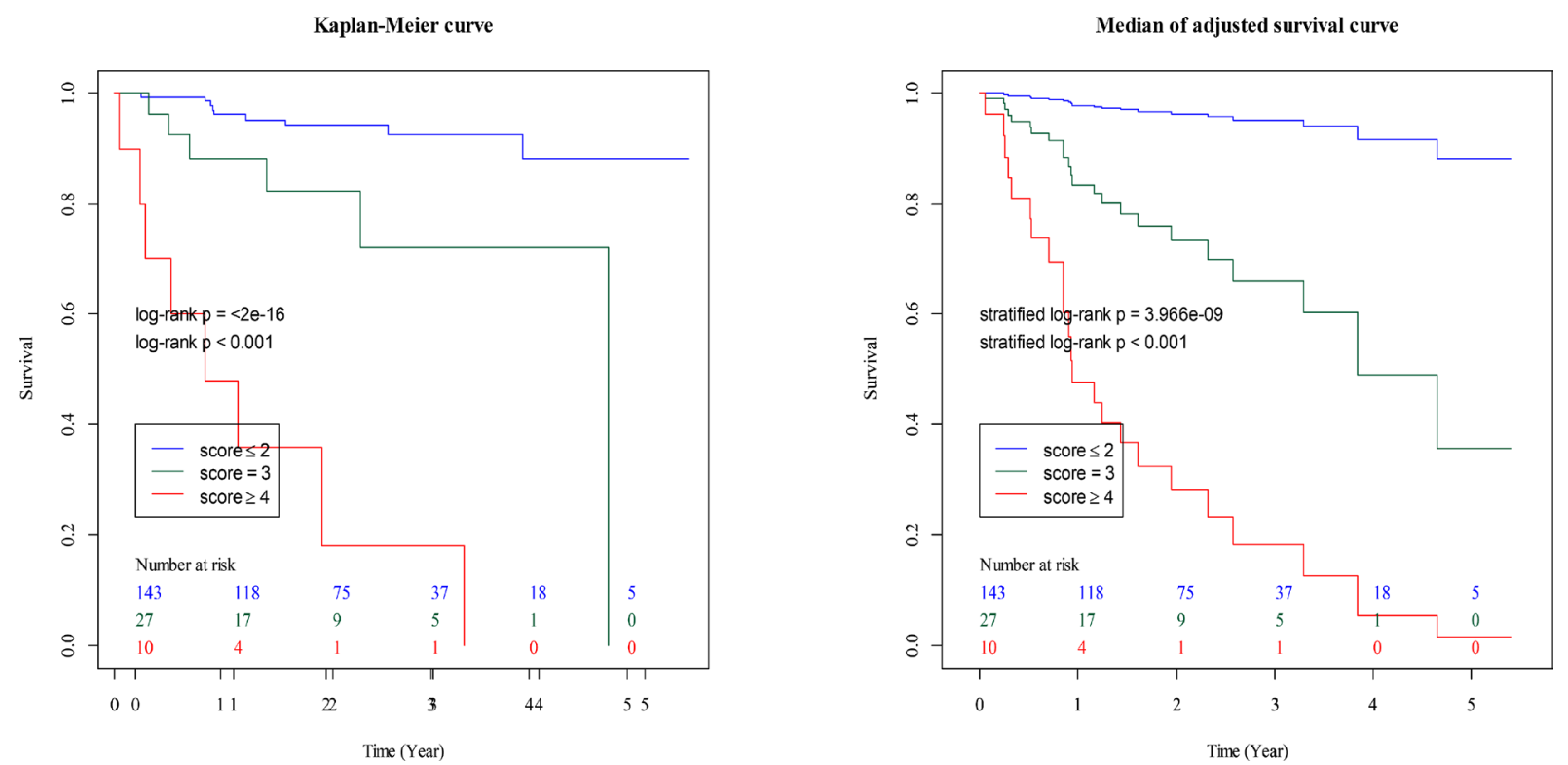

Figure S2 Comparison of patients with ILD according to composite scoring system using ILD diagnosis, CCIS, and \%FVC (score $\leq 2$ vs. $=3$ $v s . \geq 4)$. We categorized the patients having the composite score ( $\geq 3$; the latter group in Figure 3 ) into two groups: score $=3 v s$. $\geq 4$. Then we compared three-year ILD-related event-free survival rates between three groups: score $\leq 2(\mathrm{n}=143) v s .=3(\mathrm{n}=27) v s . \geq 4(\mathrm{n}=10)$. Both log-rank and stratified log-rank tests showed that Kaplan-Meier survival curves of these groups differed significantly $(\mathrm{P}<0.001)(\mathrm{A}$ : $\log$-rank test; B: stratified log-rank test). CCIS, Charlson Comorbidity Index score; ILD, interstitial lung disease; FVC, forced vital capacity.

Table S1 Scoring system including IPF diagnosis, CCIS, FVC

\begin{tabular}{lcc}
\hline Predictors & Details & Score \\
\hline The diagnosis & IPF & 1 \\
\multirow{2}{*}{ CCIS } & Not IPF & 0 \\
& $4-5$ & 4 \\
& $2-3$ & 3 \\
& $0-1$ & 2 \\
FVC, \%predicted & $<70.0$ & 1 \\
& $\geq 70.0$ & 1 \\
\hline
\end{tabular}

CCIS, Charlson Comorbidity Index score; FVC, forced vital capacity; IPF, idiopathic pulmonary fibrosis. 